\title{
Comparison of Kinematics and Muscle Activation between Push-up and Bench Press
}

\section{(ㄷ) (i) 우 $\odot$}

\author{
Author \\ Roland van den Tillaar
}

\author{
Affiliation \\ Department of Sports Sciences and Physical Education, \\ Nord University, Levanger, Norway \\ Key words \\ peak velocity, mean velocity, resistance training, strength
}

$\begin{array}{ll}\text { received } & 18.06 .2019 \\ \text { revised } & 20.07 .2019 \\ \text { accepted } & 07.08 .2019\end{array}$

\section{Bibliography}

DOI https://doi.org/10.1055/a-1001-2526

Published online: 2019

Sports Medicine International Open 2019; 3: E74-E81

(c) Georg Thieme Verlag KG Stuttgart · New York

ISSN 2367-1890

\section{Correspondence}

Prof. Roland van den Tillaar

Sports Sciences and Physical Education

Nord University

Odins veg 23

7603 Levanger

Norway

Tel.: + 47/976/62 913, Fax: + 47/7411/2 001

roland.v.tillaar@nord.no

\begin{abstract}
The purpose of this study was to compare the similarity in kinematics and upper-body muscle activation between push-up and bench press exercises over a range of loads. Twenty resistance-trained subjects (age $22.5 \pm 5.24 \mathrm{yrs}$, body mass $83.7 \pm 10.7 \mathrm{~kg}$, height $1.80 \pm 0.06 \mathrm{~m}$ ) executed bench presses and push-ups with 4 different loads. Bench press was executed at $50-80 \%$ of their assumed 1 repetition max in steps of $10 \mathrm{~kg}$, while push-ups were executed without a weight vest and with a 10-20-30 kg weight vest. A linear encoder measured kinematics (displacement, time, average and peak velocity) during the exercises at each load, together with mean and maximal muscle activation of 8 upper body muscles and their timing for each exercise and each load. The main findings of this study demonstrate no differences in kinematics and muscle activation between the two exercises and that the different loads had the same effect upon both push-up and bench press in experienced resistance-trained men. For coaches and athletes, pushups and bench presses for strength training can be used interchangeably. By using a weight vest, push-ups can mimic different loads that are similar to different intensities in the bench press that can be used to train strength demands.
\end{abstract}

\section{Introduction}

The push-up and bench press are two popular exercises used to train upper body strength $[3,16]$. The selection of which exercise to use is largely dependent on movement capabilities, the athlete's goal, relative strength levels, and the availability of equipment [10]. In bench press, a barbell and weights are necessary for training, whereas push-ups are in general performed with body weight as a load. In training, both exercises are used, but bench press is mostly used to assess maximal muscular strength [21,23], whereas push-ups are mainly used to target muscular endurance [8]. However, by using elastic bands or weight vests in push-ups, it is also possible to increase the intensity ( $>80 \%$ of 1 -repetition maximum:
1-RM) so much that it is possible to gain maximal muscular strength in advanced lifters [3]. Calatayud et al. [3] showed that by using similar loads in bench press and push-up, similar increases in strength occur. The reasoning behind this is that a similar amount of neuromuscular activation would result in similar gains.

There are only a few studies that have compared possible neuromuscular similarities and differences between the bench press and the push-up [1, 3, 10]. Although Blackard et al. [1] and Calatayud et al. [3] found no differences in neuromuscular activation between the two exercises in the long head of the triceps, pectoralis major, and anterior deltoid, Gottschall et al. [10] found that the anterior deltoid and biceps brachii were more activated during the bench press than push- 
ups. This contrast in findings may be due to the variation in the pushup exercise used. Blackard et al. [1] and Gottschall et al. [10] compared muscle activity during unloaded push-ups and equalised bench press weight to the same total load between the two exercises, whereas Calatayud et al. [3] investigated only the pectoralis major and anterior deltoid muscles during a 6-RM bench press compared with elastic band-loaded 6-RM push-ups. Thus, the equivalency of the load lifted is a limiting factor in establishing the neuromuscular differences between the exercises. All of these studies compared only one push-up load with an equalised bench press load with only the mean neuromuscular activation [10] of a limited number of muscles [1,3]. Timing of maximal activation of the muscles was not included, nor was there any kinematic analysis (velocity, displacement, movement time) to compare the specificity of the two exercises. Thus, it is not known if muscle activation and kinematics behave the same between push-ups and bench presses over a range of loads. This information is interesting for athletes, physical therapists and trainers to select or include push-ups during a strength training program, because the use of weight vests in push-ups is a feasible and cost-effective option that may be used as an alternative to the bench press exercise [3].

Therefore, the aim of this study was to investigate the similarity in kinematics and muscle activation between a bench press and a push-up over a similar range of loads. Based upon Calatayud et al. [3] and Blackard et al. [1], it was hypothesised that similar loads between the two exercises would result in similar kinematics and muscle activation.

\section{Materials and Methods}

\section{Participants}

Twenty resistance-trained subjects (age: $22.5 \pm 5.24$ yrs, body height $1.80 \pm 0.06 \mathrm{~m}$, body mass $83.7 \pm 10.7 \mathrm{~kg}$ ) participated in the study. Each participant was informed of the testing protocol and possible risks, and written consent was obtained prior to the study. The study complied with the current ethical regulations for research [12], was approved by the Regional Ethical Committee, and conformed to the latest revision of the Declaration of Helsinki.

\section{Experimental design}

To compare kinematics, muscle activation, and timing of these between push-ups and bench presses within subjects, a repeatedmeasures design was used in which all subjects conducted both exercises with four different loads in a randomised order.

\section{Procedures}

One week prior to the test session, the participants visited the laboratory for a session to become familiar with the equipment and protocol. Because participants had never before performed push-ups while wearing a weight vest, they were familiarised with using one with different weights in particular. In the test session, height and body mass were first measured before starting a general warm-up of 5 min of jogging on a treadmill, followed by several push-up attempts with the weight vest and then the bench press. After the warm-up, half of the participants were assigned to perform the bench press condition, while the other half started with the push-up condition. Each condition was performed with four different loads: the push-up without the weight vest and with the $10-20-30 \mathrm{~kg}$ weight vest (Titan Fitness, Memphis, TN, USA); and the bench press at $\sim 50-80 \%$ of the participant's self-estimated 1 repetition maximum (1-RM) in steps of $10 \mathrm{~kg}$. The different loads were given in either an increasing or decreasing order to each participant, and three repetitions per load were performed. The participants performed the bench press according to the rules and regulations set by the International Powerlifting Federation (IPF), except that they were allowed to touch and press without a full stop, but no bounce was allowed. During the bench press, each participant used their preferred grip width, which was held constant at the different loads; this width was the same as that used for the pushups, i. e., $\sim 150 \%$ of the bi-acromion distance [11]. To simulate the same depth and same grip width as in the bench press, the push-up handles used were positioned so that the grip was the same as in the bench press, and the participants had to lower their chest to the height of the push-up handles, which mimics the barbell. The test leader controlled this every time.

\section{Measurements}

The push-up handles were placed on a strain gauge force plate ( $\mathrm{Er}$ gotest Technology AS, Langesund, Norway), and sampling was performed at a frequency of $1000 \mathrm{~Hz}$. Before the participant took the push-up handles, the force plate was reset. The participant placed their feet behind the plate at the same height as the force plate. To measure the absolute load and percentage of body weight (+ weight vest) that had to be lifted during the different push-up conditions, the initial ground reaction force was used when the participant grasped the handles and was in the start position for the push-up.

A linear encoder (ET-Enc-02, Ergotest Technology AS, Porsgrunn, Norway) was attached around the subject's neck with a collar when performing a push-up and the barbell when performing bench presses, directly vertical to the ground. It measured at a resolution of $0.019 \mathrm{~mm}$ and counted the pulses with a 5 -millisecond interval vertical displacement in relation to the lowest point of the barbell (zero distance). The exercises were divided into two phases: the downward and upward phase, identified using the linear encoder. Average upward velocity was calculated from the lowest barbell position over the chest and chest to the ground in the push-up to the highest positions by using a five-point differential filter with Musclelab v10.5.67 software (Ergotest Technology AS, Porsgrunn, Norway). The maximal measurement error of velocity due to the system was less than $0.9 \%$ and the coefficient of variation was $2.3 \%$ [2]. Movement time, distance, and peak and average velocity in the downward and upward phase were measured together with the timing of maximal downward and upward velocity.

Wireless electromyography (EMG) was recorded by using a Musclelab 6000 system and analysed by Musclelab v10.5.67 software (Ergotest Technology AS, Langesund, Norway). The skin was shaved, abraded, and washed with alcohol before placement of the gel-coated self-adhesive electrodes (Dri-Stick Silver circular sEMG Electrodes AE-131, NeuroDyne Medical, USA). The electrodes ( $11 \mathrm{~mm}$ contact diameter and a $2 \mathrm{~cm}$ centre-to-centre distance) were placed along the presumed direction of the underlying muscle fibre according to the recommendations by the Surface ElectroMyoGraphy for the NonInvasive Assessment of Muscles (SENIAM) project [13, 20]. The electrodes were placed on the right upper limb positioned on the belly 
of the anterior and medial deltoid; the lateral, medial, and long head of the triceps brachii; the sternal and clavicular part of the pectoralis major; and the biceps brachii. To minimise noise from the surroundings, the raw EMG signal was amplified and filtered using a preamplifier located close to the sampling point. The EMG signals were converted to root mean square (RMS) EMG signals using a hardware circuit network (frequency response $20-500 \mathrm{kHz}$, averaging constant $100 \mathrm{~ms}$, total error $\pm 0.5 \%$ ). The peak and mean RMS EMG signals of each muscle during the upward phase of the lift with each load were used for further analysis, except for the biceps brachii in which the mean and peak activity was used of the downward phase because the greatest activity occurred in this phase [21,22]. No normalisation of the EMG signals was necessary because all measurements per participant were performed in one session and only a within-subject design was used [21,22]. In addition, the timing of peak EMG signals was collected to identify eventual differences in timing between the two exercises over the different loads. Timing was calculated as relative to the whole down/upward movement time to make it possible to compare timing over the different loads. The force plate, linear encoder and EMG were all synchronised by the Musclelab 6000 system.

\section{Statistical analysis}

To compare kinematics and muscle activation between the two exercises with the different loads, a 2 (exercise: push-up vs. bench press) $\times 4$ (load: four different loads in each exercise) analysis of variance (ANOVA) with repeated measurements was used for each of the muscles. Where the sphericity assumption was violated, we have reported Greenhouse-Geisser-corrected p-values in the results. Post hoc tests using the Holm-Bonferroni probability adjustment were used to identify differences. In addition, a 2 (exercise: push-up vs. bench press) $\times 4$ (load: four different loads in each exercise) $\times 7$ (different muscles) ANOVA with repeated measurements was used to assess the timing of the different muscles related to each other. The effect size used and reported in this study was partial eta squared $\left(\eta^{2}\right)$, where $0.01 \leq \eta^{2<0.06}$ constituted a small effect, $0.06 \leq \eta^{2}<0.14$ constituted a medium effect, and $\eta^{2}<0.14$ constituted a large effect [4]. The level of significance was set at $\mathrm{p} \leq 0.05$ for all tests and the analyses were carried out with SPSS Statistics v25 (SPSS, Inc., Chicago, IL, USA).

\section{Results}

In the 4 push-up conditions, the absolute loads that had to be lifted were from $52.3 \pm 6.8-76.7 \pm 6.8 \mathrm{~kg}$, which was from $62.6-65.1 \%$ of the subjects' body mass ( + weight vest) that had to be lifted.

No significant effect of exercise $\left(F \leq 0.25, p \geq 0.623, \eta^{2} \leq 0.01\right)$ for any of the kinematic parameters was found ( $\vee$ Fig. 1), whereas, as expected, load had a significant effect upon all kinematic variables $\left(F \leq 0.25, p \geq 0.623, \eta^{2} \leq 0.01\right)$ and no significant interaction effect $\left(F \leq 1.73, p \geq 0.19, \eta^{2} \leq 0.08\right)$. Post hoc comparisons showed that displacement, peak downward, peak and mean upward velocity decreased in both the downward and upward phases with each increasing load, whereas the mean downward velocity decreased only with the two heaviest loads ( $>$ Fig. 1). The total time in the upward phase increased with each load, whereas the time in the downward phase increased only from load 2-4 (• Fig. 1).

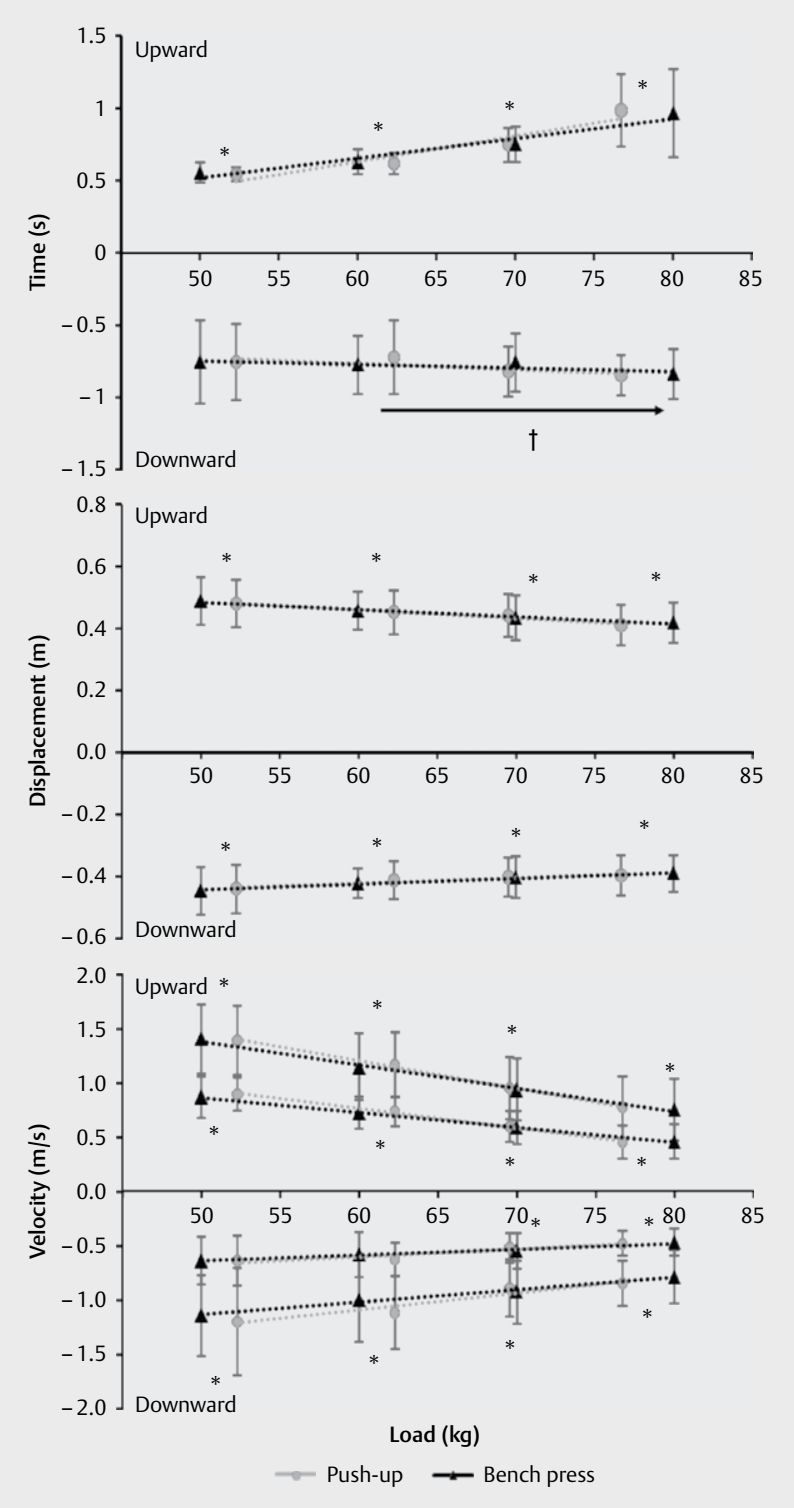

- Fig. 1 Mean ( \pm standard deviation) total time, distance, average and peak velocity downward and upward at each load of push-up and bench press averaged over all participants. * indicates a significant difference with all other loads for both exercises at the $p \leq 0.05$ level. $\dagger$ indicates a significant difference between these two loads for both exercises at the $p \leq 0.05$ level.

Also, no significant effect of exercise $\left(F \leq 2.53, p \geq 0.128, \eta^{2} \leq 0.11\right)$ was found for any of the mean and maximal muscle activities ( $>$ Fig. 2). A significant effect of load ( $F \geq 4.08, p \leq 0.031, \eta^{2} \geq 0.18$ ) was found for the mean muscle activities of the sternal part of the pectoralis major, anterior deltoid, and the long head of the triceps brachii, together with the peak muscle activation of the long head of the triceps brachii. For the other muscles, no significant effect of load was found $(F \leq 2.49$, $\left.p \geq 0.114, \eta^{2} \leq 0.11\right)$, nor was any significant interaction effect observed $\left(F \leq 1.95, p \geq 0.151, \eta^{2} \leq 0.11\right)$. Post hoc comparisons showed that only mean muscle activity for the sternal part of the pectoralis major increased only between loads 2 and 3, for the anterior deltoid between loads 2 and 4 , and for the maximal activity of long head of triceps bra- 

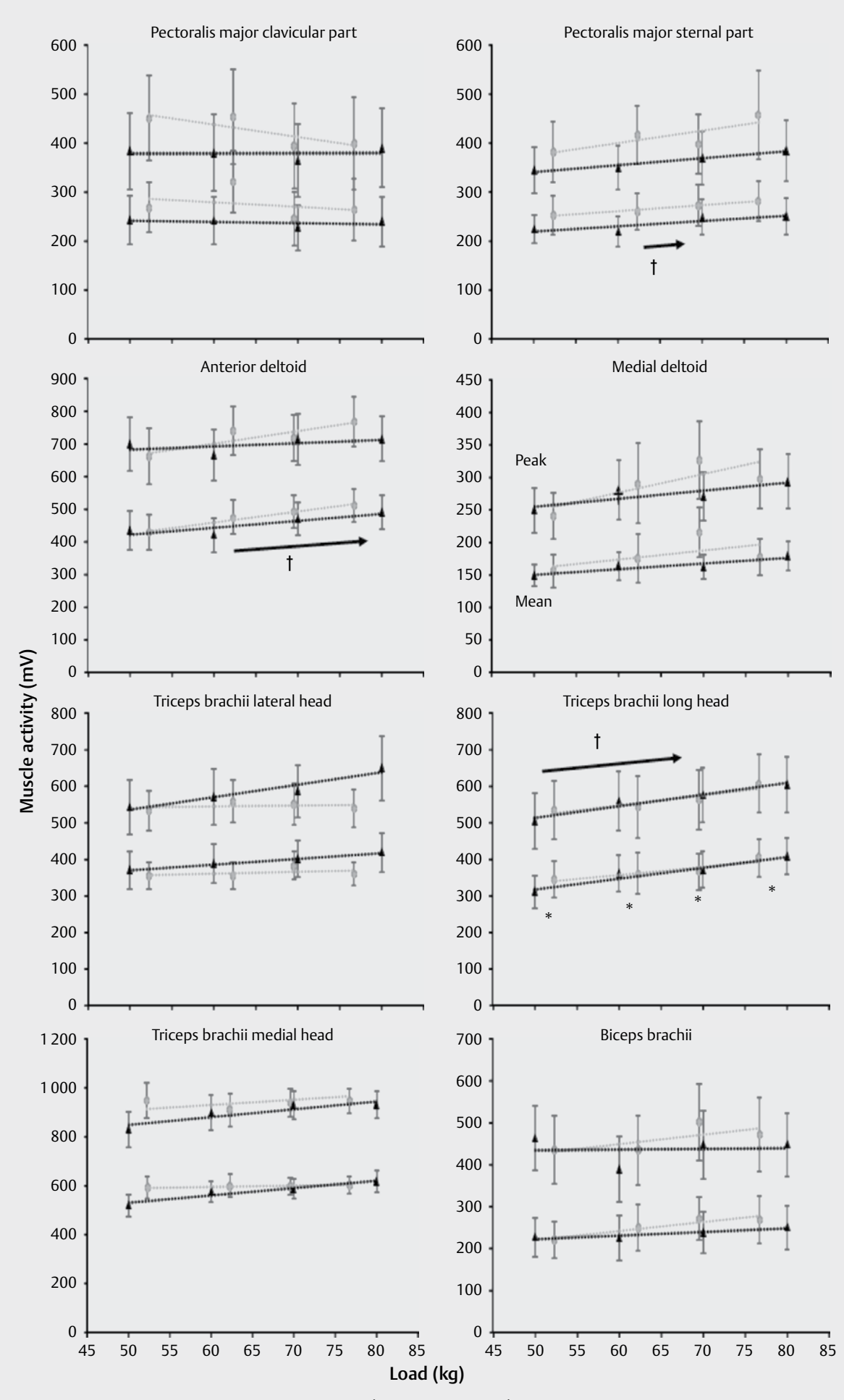

$\longrightarrow$ Push-up

Bench press

- Fig. 2 Mean ( \pm standard error of the mean) and peak muscle activation of all muscles during the upward phase (biceps brachii activation in downward phase) of the lifts at each load and exercise averaged over all participants. . ${ }^{*}$ indicates a significant difference in muscle activation with all other loads for both exercises at the $\mathrm{p} \leq 0.05$ level. $\rightarrow$ indicates a significant increase in muscle activation from this load to all those to the right of the arrow at the $\mathrm{p}<0.05$ level.

chii between loads 1 and 3. The mean muscle activity of the long head of the triceps brachii increased significantly for every load ( $\vee$ Fig. 2 ).
A significant effect in the timing of peak downward and upward velocity $\left(F \geq 4.34, p \leq 0.024, \eta^{2} \geq 0.19\right)$ was found. Furthermore, the timing of peak muscle activation between muscles $(F=4.16, p=0.001$, 
$\left.\eta^{2}=0.20\right)$ and load $\left(F=18.0, p<0.001, \eta^{2}=0.51\right)$ was significant, but no significant effect of exercise $\left(F=0.09, p=0.762, \eta^{2}<0.01\right)$ or any interaction effects $\left(F \leq 2.35, p \geq 0.099, \eta^{2} \leq 0.12\right)$ were found. When the effect of load per muscle was evaluated, a significant effect was found for all muscles except the biceps brachii $(p=0.698)$ and medial deltoid $(p=0.50)$ ( $\triangleright$ Fig. 3). Post hoc comparisons revealed that, when timing was averaged over all loads and exercises, peak muscle activation occurred at almost the same time for all muscles, except for the triceps medial head in which peak activation occurred significantly later than in all other muscles except the long head of the triceps. Furthermore, the anterior deltoid and lateral triceps brachii peak activation occurred significantly earlier than the peak activation of the long head of the triceps brachii ( $\vee$ Fig. 4). When viewed per condition and load, the timing of peak activation occurred significantly later in the lift from load 1-2 (pectoralis, anterior deltoid, and lateral triceps brachii) and from load 2-3 (other two triceps muscles). Also, a significant increase from load 3-4 was found for the sternal part of the pectoralis major. The timing of peak downward velocity occurred earlier, whereas the peak upward velocity occurred later with increasing loads ( Fig. 3).

\section{Discussion}

The purpose of this study was to investigate if kinematics and muscle activation are similar between bench press and push-up exercises over a similar range of loads. The main findings of this study demonstrate no differences in kinematics and muscle activation between the two exercises and that the different loads had the same effect upon both push-up and bench press exercises.

As expected, the displacement and the mean and peak velocity decreased, and movement times increased in both phases for both exercises with no differences between the two exercises ( $\bullet$ Fig. 1). That there was no difference in kinematics between the two exercises is explainable by the fact that there were no neuromuscular differences in any of the measured muscles between the two exercises, indicating that similar loads result in similar neurological responses for push-ups and bench presses. This was also found by Blackard et al. [1] and Calatayud et al. [3], who compared only one load (unweighted push-ups) between the two exercises. In the present study, over the whole range of external loads, the kinematics and muscle activation between the two exercises behaved the same, indicating that push-ups with weight vests can be used interchangeably with the bench press to enhance upper body strength. Earlier it was suggested that because push-ups are seen as a closed kinetic chain exercise and the bench press as an open kinetic chain exercise $[1,5]$, the result would be different muscle activity between the two exercises owing to different stability concerns in the distal segments [6]. In an open kinetic chain exercise (bench press), the distal segment, in this case the hands, is not fixed to the floor and therefore would require more muscle activity to stabilise the load on the end of the segment than in a closed kinetic chain like push-ups [6]. However, as indicated by Dillman and Murray [5], if the direction and mass of loading in both exercises are similar, the electromyographic activity of the primary muscle groups involved would be comparable. In the present study, the kinematics were the same, thus muscle activation would also be the same, as shown.
Kinematics changed mostly in the upward phase with increasing external load, which is in accordance with Gonzalez-Badillo and Sanchez-Medina [9] and Newton et al. [18], who showed that peak and average velocity decreased with increasing load. However, the total distance down and upwards declined with increasing external load ( $\triangleright$ Fig. 1), indicating that the load also affected the starting position. The difference was only $0.04 \mathrm{~m}$ between the lifts with the lowest and heaviest loads. These differences were probably caused by not lowering the trunk or barbell fully to avoid losing control at the lowest point or had been caused by the large loads not allowing for full shoulder protraction at the start of the movements. This was also shown by the earlier occurrence of peak downward velocity with increasing load ( $\vee$ Fig. 3). Furthermore, the upward distance was on average $0.04 \mathrm{~m}$ longer than the downward phase, which indicates that the participants performed this upward phase with full effort. The longer movement time upward is also visible in relatively later occurrence of peak muscle activation of most muscles with increasing load ( $\vee$ Fig. 3), which was probably caused by the existence of a sticking region [7]. A sticking region is a region in which velocity decreases after the initial peak velocity and occurs with increasing load. It is the weakest point during the upward phase of a lift $[7,22]$. Velocity increases again once the sticking point is passed.

The timing of maximal peak activation of most muscles in both exercises occurred at around $30-40 \%$ of the upward phase of the lift, which is around where the sticking point is. To pass this point, which is the weakest point of the lift, it is important to have maximal activation of the muscles. The later occurrence of the peak activation of the medial head of the triceps was probably caused by the fact that this muscle, together with the long head, are only moderately pennated and thereby less strong than the lateral head of the triceps, which reaches its peak activation earlier [15]. Furthermore, due to the origin of the long head of the triceps on the intraglenoid tubercle, this muscle could also cause a shoulder extension moment, especially when active at the lowest point of the lift. Therefore, peak activation of this muscle has to occur later because it works more as a shoulder stabiliser [14].

With increasing load from $\sim 50-80 \%$, not all muscles increased their neuromuscular activation. It seems that mainly the long head of the triceps brachii, the anterior deltoid and the sternal part of the pectoralis major significantly increased their activation $[17,18]$. These muscles are responsible for overcoming the external load with increasing demands. These muscles are also the limiting factor in maximal bench press lifting $[22,24]$, whereas other muscles are more accountable for stability [19].

Some limitations to the present study are that only loads between 50 and $80 \mathrm{~kg}$ were used and not maximal loads. However, it is difficult to get weight vests for this population that are heavy enough to reach maximal loading. Furthermore, in the present study, activation was measured in only a few muscles that are mainly responsible for elbow extension and shoulder flexion; no muscles that stabilise the trunk and pelvis were assessed. Gottschall et al. [10] showed that the rectus abdominis has a significantly higher activation during a push-up than a bench press, with the explanation that the trunk is supported during the open kinetic chain exercise (bench press), so there is little need for core activation. Based on this finding, Gottschall et al. [10] suggested that push- 

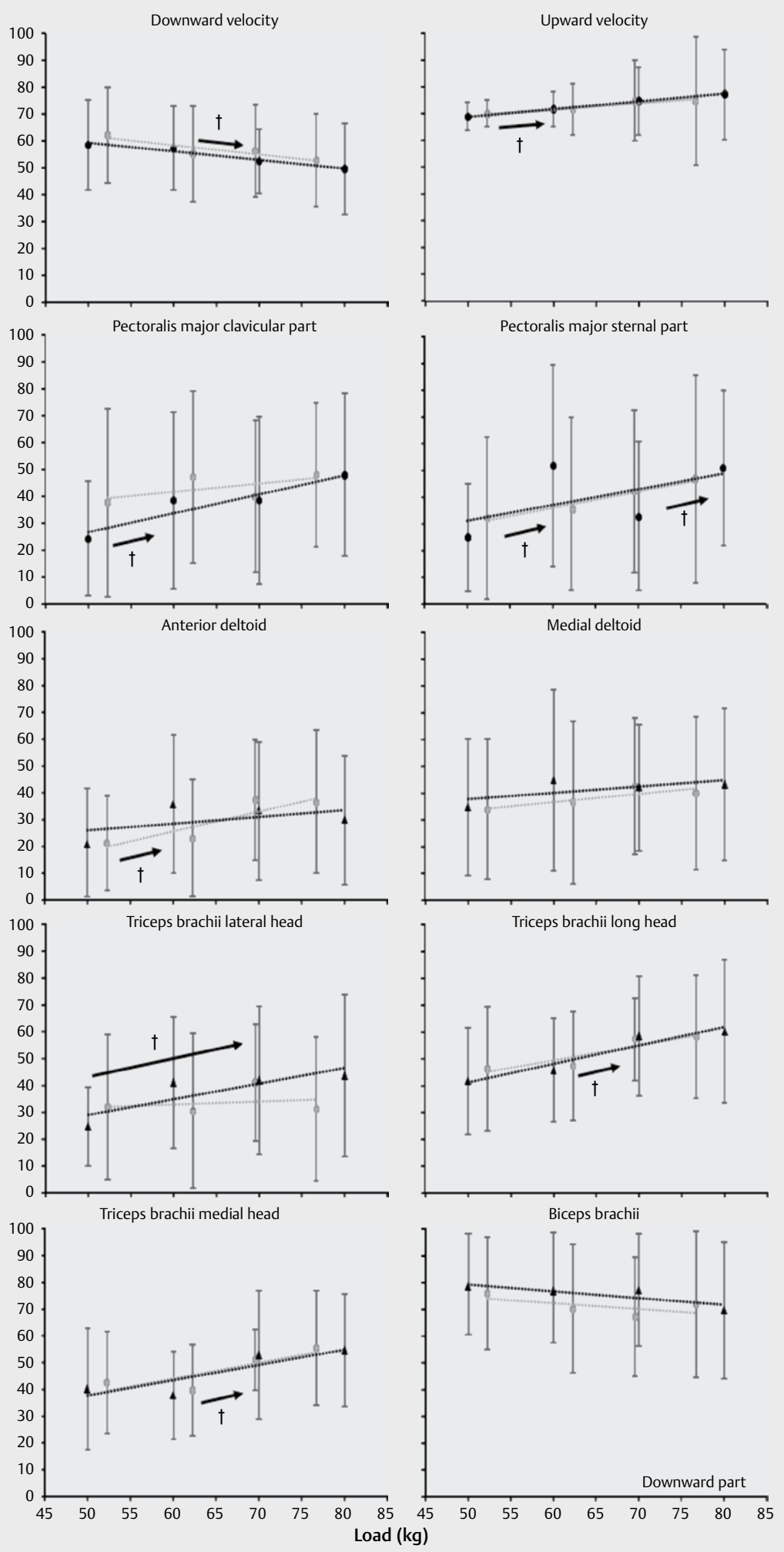

$-\rightarrow-$ Push-up $\quad \mathbf{t}-$ Bench press

- Fig. 3 Mean ( \pm standard deviation) timing of occurrence of peak muscle activation of all muscles during the upward phase (biceps brachii activation in downward phase) of the lifts at each load and exercise averaged over all participants. $\rightarrow$ indicates a significant change in timing of occurrence of peak muscle activation from this load to all those to the right of the arrow at the $p<0.05$ level. 


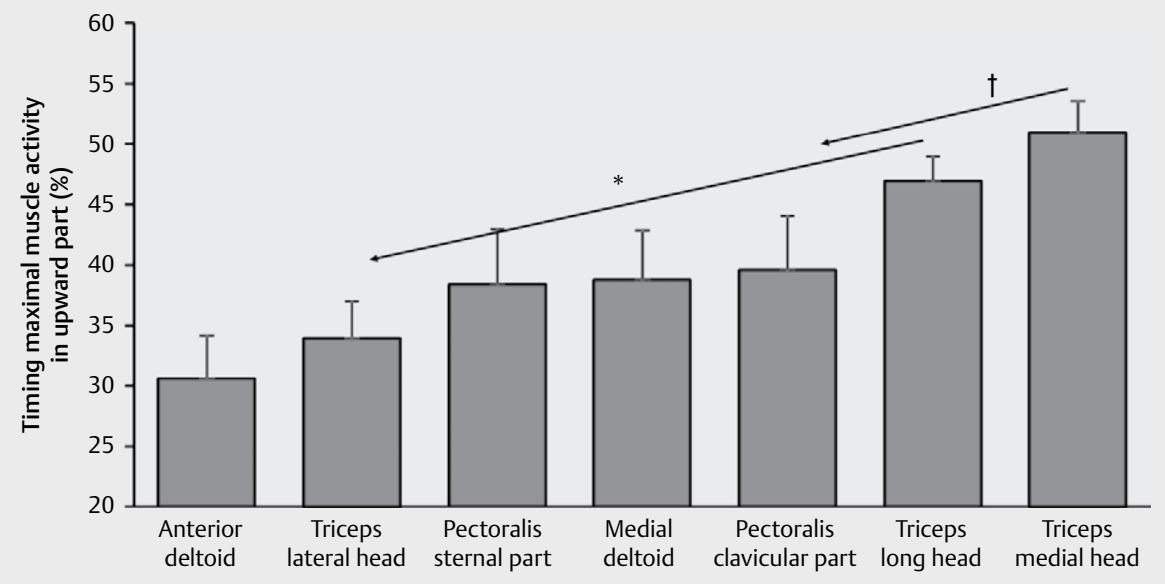

- Fig. 4 Mean ( \pm standard error of the mean) timing of peak muscle activation of the muscles during the upward phase when averaged over all loads, exercises, and all participants. $\leftarrow$ indicates a significant change in timing of occurrence of peak muscle activation between this muscle and all those to the left of the arrow at the $p<0.05$ level.

ups (closed kinetic chain exercise) may be more effective for integrative or functional movement training if the athlete is strong enough to execute a normal push-up. Thus, when the level of the athlete is not high enough ( $<63 \%$ of body mass) to perform a normal push-up, the bench press could be used to increase strength. However, when the strength is high enough, both bench press and/ or push-ups with a weight vest or other extra resistance (e. g. combined with elastic bands) to mimic similar loads can be used interchangeably to increase strength [3]. Push-ups with a weight vest are recommended because they also target the core muscles and are easier and cheaper to use in training.

\section{Conclusion}

Based upon the findings of the present study, we can conclude that no differences in kinematics and muscle activation were found between the bench press and push-ups over a range of loads in experienced resistance-trained athletes, and that the different loads had the same effect upon both push-up and bench press. Therefore, it is possible to use the bench press and loaded push-up interchangeably when training the upper body.

\section{Acknowledgements}

Thanks go to Sigve Knarbakk for the data collection and to the participants who contributed to this study. This study was conducted without any funding from companies, manufacturers or outside organisations.

\section{Conflict of Interest}

The authors declare that they have no conflict of interest.

\section{References}

[1] Blackard DO, Jensen RL, Ebben WP. Use of EMG analysis in challenging kinetic chain terminology. Med Sci Sports Exerc 1999; 31: 443-448

[2] Bosco C, Belli A, Astrua M, Tihanyi ], Pozzo R, Kellis S, Tsarpela O, Foti C, Manno R, Tranquilli C. A dynamometer for evaluation of dynamic muscle work. Eur J Appl Physiol Occup Physiol 1995; 70: 379-386

[3] Calatayud J, Borreani S, Colado JC, Martin F, Tella V, Andersen LL. Bench press and push-up at comparable levels of muscle activity results in similar strength gains. J Strength Cond Res 2015; 29: 246-253

[4] Cohen J. Statistical Power Analysis for the Behavioral Sciences. $2^{\text {nd }}$ ed. Hillsdale, NJ: Lawrence Erlbaum Associates; 1988; 174

[5] Dillman C], Murray TA. Biomechanical differences of open and closed chain exercises with respect to the shoulder. J Sport Rehab 1994; 3: 228-238

[6] Duscha BD, Cipriani DJ, Roberts CP. A review of open vs. closed kinetic chain exercise for lower extremity rehabilitation. Clin Exerc Physiol 1999; 1: 57

[7] Elliott BC, Wilson G], Kerr GK. A biomechanical analysis of the sticking region in the bench press. Med Sci Sports Exerc 1989; 21: 450-462

[8] Garcia-Masso X, Colado JC, Gonzalez LM, Salva PAU, Alves J, Tella V, Triplett NT. Myoelectric activation and kinetics of different plyometric push-up exercises. J Strength Cond Res 2011; 25: 2040-2047

[9] Gonzalez-Badillo J], Sanchez-Medina L. Movement velocity as a measure of loading intensity in resistance training. Int J Sports Med 2010; 31: 347-352

[10] Gottschall JS, Hastings B, Becker Z. Muscle activity patterns do not differ between push-up and bench press exercises. J Appl Biomech 2018; 34: 442-447

[11] Gouvali MK, Boudolos K. Dynamic and electromyographic analysis in variations of push-up exercise. J Strength Cond Res 2005; 19: 146-151

[12] Harriss DJ, Macsween A, Atkinson G. Standards for ethics in sport and exercise science research: 2018 update. Int J Sports Med 2017; 38: 1126-1131

[13] Hermens HJ, Freriks B, Disselhorst-Klug C, Rau G. Development of recommendations for SEMG sensors and sensor placement procedures. J Electromyogr Kinesiol 2000; 10: 361-374 
[14] Landin D, Thompson M. The shoulder extension function of the triceps brachii. J Electromyogr Kinesiol 2011; 21: 1601-1605

[15] Langenderfer J, Jerabek SA, Thangamani VB, Kuhn JE, Hughes RE. Musculoskeletal parameters of muscles crossing the shoulder and elbow and the effect of sarcomere length sample size on estimation of optimal muscle length. Clin Biomech 2004; 19: 664-670

[16] Mayhew JL, Ball TE, Arnold MD, Bowen JC. Push-ups as a measure of upper body strength. J Appl Sport. Sci Res 1991; 5: 16-21

[17] McCaw ST, Friday JJ. A comparison of muscle activity between free weight and machine bench press. J Strength Cond Res 1994; 8: 259-264

[18] Newton RU, Murphy AJ, Humphries B], Wilson G], Kreamer W], Häkkinen K. Influence of load and stretch shortening cycle on the kinematics, kinetics and muscle activation that occurs during explosive upper-body movements. Eur J Appl Physiol 1997; 50: $311-320$
[19] Saeterbakken A, van den Tillaar R, Fimland M. A comparison of muscle activity and 1 -RM strength of three chest-press exercises with different stbility requirements. J Sports Sci 2011; 29: 533-538

[20] Saeterbakken AH, Fimland MS. Muscle force output and electromyographic activity in squats with various unstable surfaces. J Strength Cond Res 2013; 27: 130-136

[21] van den Tillaar R, Ettema G. A comparison of successful and unsuccessful attempts in maximal bench pressing. Med Sci Sports Exerc 2009; 41: 2056-2063

[22] van den Tillaar R, Ettema G. The "sticking period" in a maximum bench press. J Sports Sci 2010; 28: 529-535

[23] van den Tillaar R, Saeterbakken A. Effect of fatigue upon performance and electromyographic activity in 6-RM bench press. J Hum Kin 2014; 40: $57-65$

[24] van den Tillaar R, Saeterbakken AH, Ettema G. Is the occurrence of the sticking region the result of diminishing potentiation in bench press? J Sports Sci 2012; 30: 591-599 